\title{
Otoacoustic Interrelationships of the Barn Owl
}

\author{
Christopher Bergevin*, Geoffrey A. Manley ${ }^{\dagger}$ and Christine Köppl ${ }^{\dagger}$ \\ * Department of Physics \& Astronomy, York University, Canada \\ ${ }^{\dagger}$ Cluster of Excellence "Hearing4all", Research Center Neurosensory Science, and Department of \\ Neuroscience, Oldenburg University, Germany
}

\begin{abstract}
.
Significant debate still exists about the biophysical mechanisms at work in otoacoustic emission (OAE) generation and how such may differ between mammals and non-mammals given gross morphological differences (e.g., existence of basilar membrane traveling waves, degree of tectorial membrane coupling). To further elucidate general principles at work, we examined the barn owl for interrelationships between spontaneous emissions (SOAEs) and those evoked using a single tone (SFOAEs). First, most ears exhibited SOAEs as a stable periodic 'rippling' whose peak-to-peak spacing was relatively constant $(\sim 0.4 \mathrm{kHz})$. Some ears showed substantially larger narrowband peaks, although their statistical distributions were highly noisy. Second, significant interactions between a low-level tone and SOAE activity were observed via an interference pattern as the tone frequency was swept. Using a suppression paradigm to extract SFOAEs as the residual, the magnitude exhibited a stable pattern of peaks and valleys unique to each ear. Third, SFOAE phase exhibited significant accumulation as frequency was swept, with a phase-gradient delay of approximately $2 \mathrm{~ms}$ that was constant across frequency. The amount of SFOAE phase accumulation between adjacent SOAE peaks tended to cluster about an integral number of cycles, as previously observed for humans. Taken together, our data suggest that the principles underlying how active hair cells work together (e.g., entrainment, phase coherence) are shared between widely different inner ear morphologies, leading to the generation of OAEs with similar properties.
\end{abstract}

\section{INTRODUCTION}

Spontaneous otoacoustic emissions (SOAEs) are commonly regarded as evidence for an 'active ear', yet are relatively uncommon in mammals. Barn owls present an interesting opportunity for study as they have an inner ear morphology differing substantially to that of mammals [6], exhibit strong OAE activity [4, 17], and have well-characterized neurophysiology (e.g., known tonotopic map) [10,11] and psychophysics [5].

Several theoretical studies have examined SOAE generation using a variety of biomechanical foundations (e.g., $[14,18])$. Motivated by morphological differences in birds relative to mammals (see [12]), such as hair cell arrangement [6], tectorial membrane coupling [16], and the uncertain role of basilar membrane traveling waves [7], our present goal was to better elucidate general biophysical characteristics of the underlying active elements and their interactions. To this end, we systematically explored interrelationships within individual barn owl (Tyto alba) ears between SOAEs and stimulus frequency emissions (SFOAEs) evoked by a single, low-level tone.

\section{METHODS}

Measurements were made in 9 lightly anesthetized (ketamine/xylazine) owls with a vented middle ear. Animals were warmed by a heating blanket. An Etymotic ER-10C probe was coupled to the ear canal. SFOAEs were evoked by swept tones, generally between 1 and $11 \mathrm{kHz}$, and extracted using a suppression paradigm [9]. Probe levels varied from 0-50 dB SPL, with a suppressor level $\left(L_{\mathrm{S}}\right) 15 \mathrm{~dB}$ higher and $40 \mathrm{~Hz}$ higher in frequency. SOAE activity was analyzed by a variety of methods (see Results). Sample rate for all data acquisition was $44.1 \mathrm{kHz}$. Additional methodological details are similar to those described previously (e.g., [3, 15]).

\section{RESULTS}

\section{SOAEs \& Effects of an external tone}

Spontaneous emissions were observable in all ears examined. At a minimum, a rippling pattern atop the microphone noise floor was apparent. This rippling had a periodic structure to it, with a uniform spacing between maxima. In the 
A
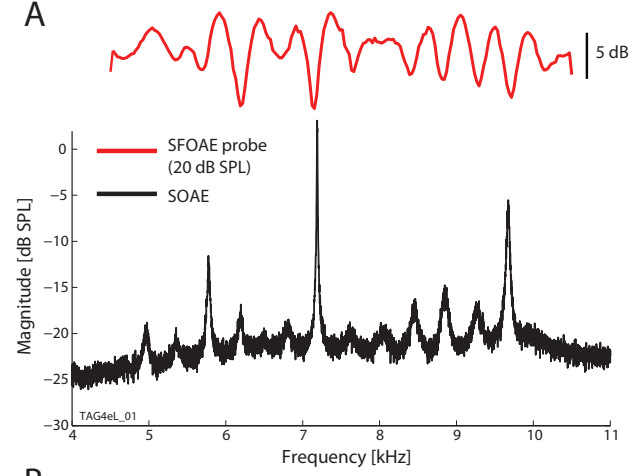

B

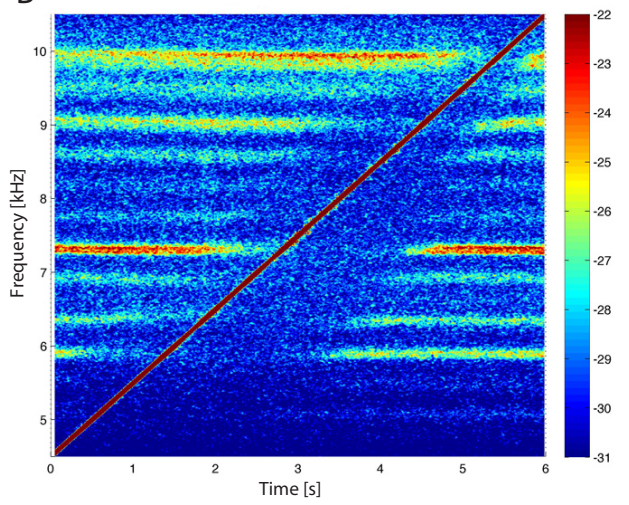

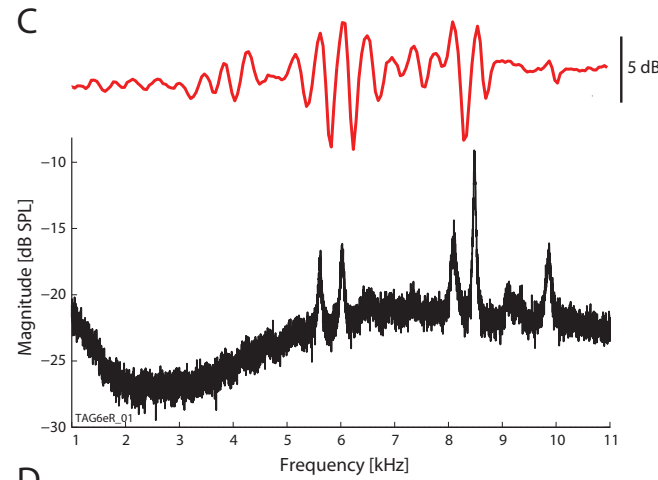

D

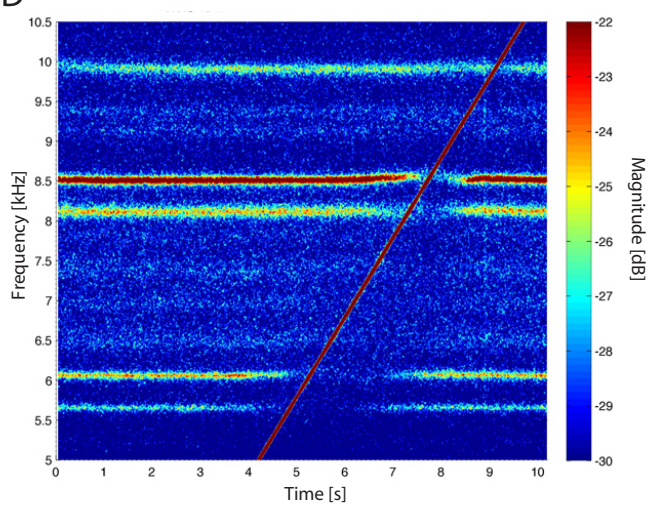

FIGURE 1. Barn owl SOAE spectra and the effects of presenting a $20 \mathrm{~dB}$ SPL tone. Data are shown from two different representative animals (each column). The top row (A and C) shows the SOAE spectra (black curves; collected in the absence of any stimuli just prior to the SFOAE run) and the interference apparent at the external auditory meatus upon presentation of the $20 \mathrm{~dB}$ SPL tone (red curve). The red curves have been offset vertically for improved visualization. The SOAE spectra were obtained by spectrally averaging 60 buffers of 32768 points each. The spectrograms shown in the bottom row (B and D) show the effect of the probe tone (apparent as the diagonal trace) as it was swept across frequency. These were obtained from the 'probe alone' segment of the SFOAE measurements by using a 4096 point window with sliding overlap of 97\% (yielding frequency and temporal resolutions of $\sim 11 \mathrm{~Hz}$ and $\sim 3 \mathrm{~ms}$ respectively) and then averaging for the $\sim 30$ sweeps collected during a given SFOAE run. Note that SOAE peaks appear as horizontal bands.

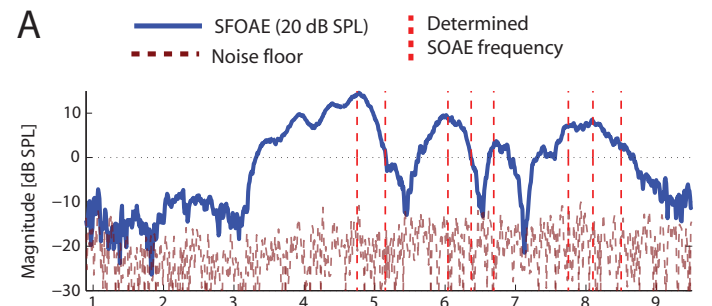

B
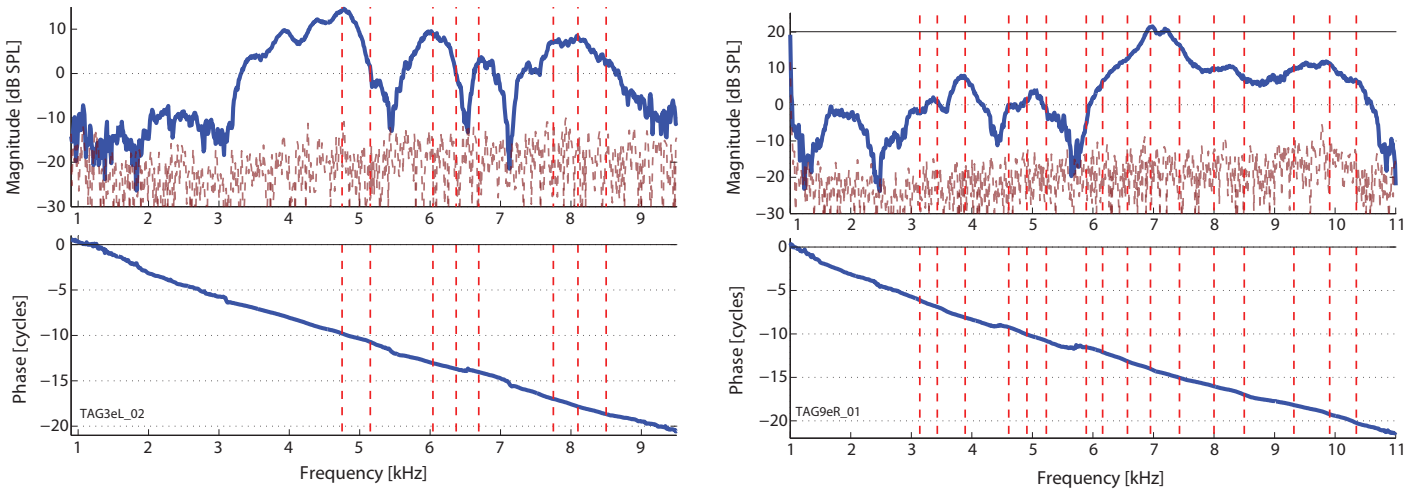

FIGURE 2. Barn owl SFOAEs and the associated SOAE peak locations $\left(L_{\mathrm{p}}=20 \mathrm{~dB}\right.$ SPL). Data are shown from two different representative animals, magnitudes in the top row and phase in the bottom. Also shown are the SOAE peak frequencies (vertical red dashed lines) extracted from the SFOAE waveforms (see text). Note that for the animal shown in B, the SFOAE magnitude actually exceeded the stimulus level when close to a large SOAE peak $(\sim 7 \mathrm{kHz})$. 
majority of ears, at least one or more large distinct peaks were observed. Most SOAE peaks exhibited statistical distributions consistent with filtered noise (not self-sustained sinusoids) [4], even for those with large SNRs.

A single probe tone had two salient effects when swept across frequency, as measured at the external auditory meatus. First, there was a strong oscillatory pattern to the probe pressure (red curves in Figs.1A and C). Presumably this is due to interference between the probe (i.e., stimulus) tone and (relatively delayed) evoked emission from the ear $[13,15,20]$. Second, as apparent in Figs.1B and D, the tone created a localized suppressive effect. Since SOAE peak frequencies could vary somewhat throughout an experiment, the most reliable way to quantify SOAE peak location was during the SFOAE sweeps themselves. As apparent in Fig.1B and D, the probe tone was swept slowly enough such that only a localized band of 'suppression' was apparent; outside that time interval the SOAE structure is stable. A $2 \mathrm{~dB}$ SNR criterion was used to qualify SOAE peaks, which corresponds visually to the appearance of horizontal bands in Fig.1B and D. This identification scheme was further validated by examining long time waveforms (120 s) collected before or after the SFOAE run where more spectral averaging would emphasize the rippling/peak structure hinted at in Fig.1. The ears shown in Fig.1 were determined to have 12 and 10 SOAE peaks respectively. Altogether, 115 peaks were identified across 15 unique ears examined. The mean/standard deviation inter-peak spacing using this approach was $0.45 \pm 0.24 \mathrm{kHz}$, suggestive of a delay of $\sim 2.2 \mathrm{~ms}$.
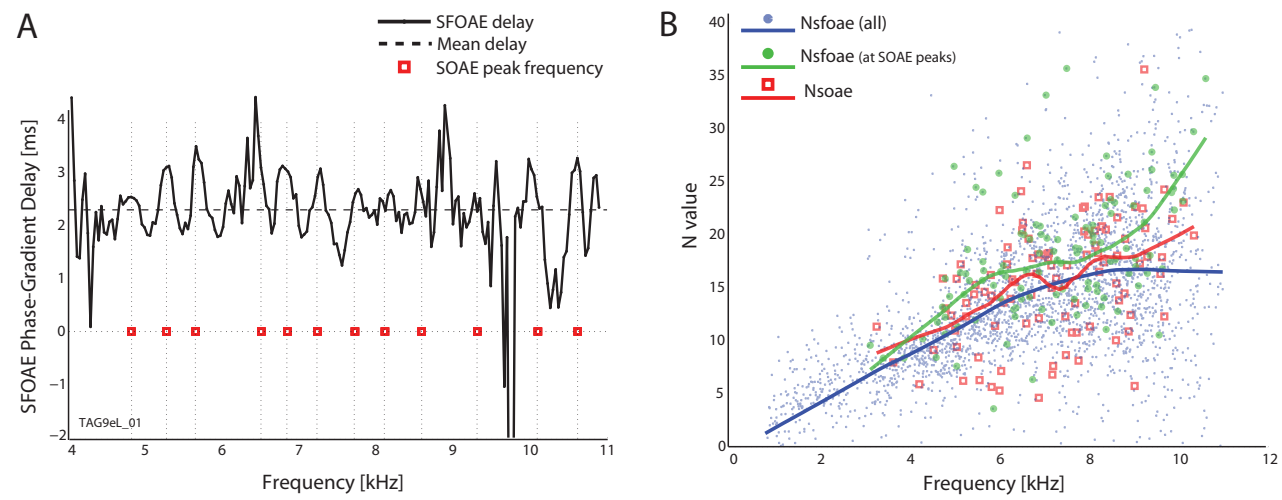

FIGURE 3. Variations in SFOAE phase-gradient delays $\left(L_{\mathrm{p}}=20 \mathrm{~dB}\right.$ SPL). Part A shows data from a representative ear, the red squares and vertical dashed lines indicating the extracted SOAE peak frequencies. The black dashed line shows the mean delay for the frequency range shown. Part B shows the various $N$ values (see text). For $N_{\mathrm{SFOAE}}$, data are shown in two ways: all values (blue) and only points whose frequency was at (or just below) a specified SOAE peak (green). Trend lines (locally-weighted regression) are included for visual clarity.
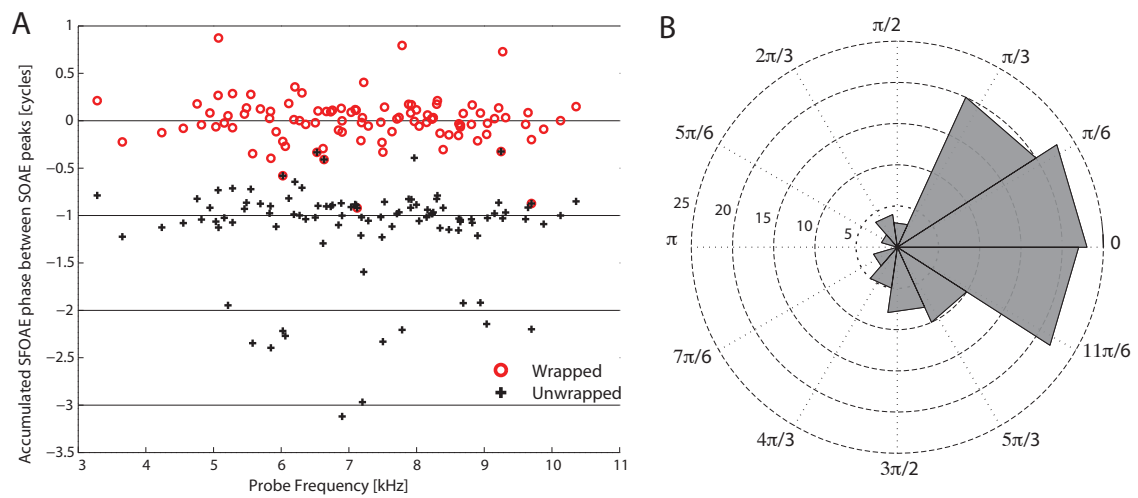

FIGURE 4. SFOAE phase accumulation between adjacent SOAE peaks ( $L_{\mathrm{p}}=20 \mathrm{~dB}$ SPL). Data are pooled across all animals (9) and ears (15) examined, for a total of 115 SOAE peaks. Data are plotted in two different ways. On the left (A), the total amount of phase accumulation (plotted versus the geometric mean frequency of the two adjacent SOAE peaks) is shown for both the SFOAE phase wrapped (red $\circ$ ) and unwrapped (black + ). The right (B) shows a polar histogram for the unwrapped condition modulus 1 cycle. Both plots show that the phase accumulation tended to cluster about an integral number of cycles. 


\section{SFOAEs \& phase accumulation between SOAE peaks}

Consistent with interference patterns observed in the probe signal (red traces shown in Figs.1A and C), all ears examined exhibited robust SFOAE activity regardless of the degree of SOAE activity observed. Focusing here on SFOAEs evoked using a probe level $\left(L_{\mathrm{p}}\right)$ of $20 \mathrm{~dB}$ SPL, Fig.2 shows responses from a single ear of two different individuals. SFOAE magnitudes showed an irregular, but stable/repeatable, pattern unique to a given ear. In general, SOAE peaks frequencies corresponded to SFOAE maxima, though this was not always the case. SFOAE phase showed significant accumulation, indicative of a delay. As shown in Fig.3A, the SFOAE phase-gradient delay was, to first order, relatively constant across frequency and typically about $2 \mathrm{~ms}$ (similar to that inferred from the SOAE spacing). However, localized variations were apparent, typically with maxima about the location of SOAE peaks. SFOAE delays are also plotted as the number of stimulus periods $\left(N_{\mathrm{SFOAE}}\right.$, Fig.3B). While not described here in detail, we note that varying the probe level (0-50 dB SPL) revealed frequency-dependent nonlinear aspects of SFOAE growth, with nonmonotonic regions typically corresponding to areas about pronounced SOAE activity. SFOAE phase-gradient delays appeared roughly constant with respect to $L_{\mathrm{p}}$ at lower levels ( $\leq 30 \mathrm{~dB}$ SPL), but decreased above moderate levels $(>30$ $\mathrm{dB}$ SPL). Further study is needed in this regard.

SFOAE phase accumulation between adjacent SOAE peaks is shown in Fig.4. Accumulation tended to cluster about integral numbers of cycles (typically one), regardless of whether the SFOAE phase had been unwrapped or not. This effect was robust: adding a small dither (i.e., $10-20 \mathrm{~Hz}$ ) to the specified SOAE frequencies did not strongly affect this clustering. This effect is similar to that previously observed for humans [1]. The relationship between SFOAE phasegradient delays and SOAE peak spacing can be seen in Figs.3B. For SOAEs, the value $N_{\text {SOAE }}$ represents the geometric mean frequency of an SOAE peak pair divided by their frequency separation [14]. In general, $N_{\mathrm{SOAE}}$ and $N_{\mathrm{SFOAE}}$ were proportional, though $N_{\text {SOAE }}$ was typically larger than $N_{\text {SFOAE }}$. However, when only $N_{\text {SFOAE }}$ values at SOAE peak frequencies are considered (green group), a different relationship between $N_{\mathrm{SFOAE}}$ and $N_{\mathrm{SOAE}}$ can be observed.

\section{DISCUSSION}

\section{Interpretation within different classes of theoretical models}

In light of the similarities with regard to human SOAE/SFOAE interrelationships and difference with respect to cochlear morphology, the barn owl data further motivate the question [14]: Does the biophysical basis for phase interactions and the longitudinal coupling of energy differ substantially across morphologies? The 'global standing wave' model [14] proposed that the appearance of 'standing waves' need not necessarily be dependent upon traveling waves along the basilar membrane per se, but could arise via other mechanisms that give rise to the appropriate phase differences (e.g., delays due to tuning). Such a conjecture was supported by models of the lizard ear [2, 19] and may apply to the owl as well. These observations suggest that the general biophysical principles determining how individual hair cells collectively behave together are similar across vertebrate ears despite gross morphological differences that would affect their coupling.

\section{Phase interactions between oscillators}

Assuming that each hair cell can be considered as a unique limit cycle oscillator (e.g., [8]), the morphology of the bird inner ear $[6,16]$ suggests that a strong degree of coupling would be present between hair cells. As shown in Fig.3, SFOAE phase-gradient delays showed localized increases about SOAE peaks. This is likely a telling point: Does this represent a phase coherence (i.e., synchronization) effect from individual oscillators contributing to a given peak and their entrainment dynamics to the probe tone? And what physical considerations ultimately determine the effective tuning of an individual oscillator? One area that needs further clarification is the basis for clustering both in the presence and absence of external stimuli. For example, small SOAE ripples can still be correlated to large SFOAE magnitudes, suggesting that SOAE peaks are more of a special case and do not completely represent how the underlying oscillators react to external stimuli. Presumably related to this point is the observation that barn owl SOAE peaks tend to have relatively noisy amplitude distributions [4]. Further study in non-mammals, such as lizards, where there is a wide diversity in tectorial membrane structure [3,12], should help resolve these questions.

\section{ACKNOWLEDGMENTS}

Supported by the Deutsche Forschungsgemeinschaft (CRC/TRR 31 "Active Hearing") to CK and Natural Sciences and Engineering Research Council of Canada (NSERC) to CB. 


\section{REFERENCES}

[1] Bergevin C, Fulcher A, Richmond S, Velenovsky D, Lee J (2012) Interrelationships between spontaneous and low-level stimulus-frequency otoacoustic emissions in humans. Hear Res 285:20-28

[2] Bergevin C, Shera CA (2010) Coherent reflection without traveling waves: On the origin of long-latency otoacoustic emissions in lizards. J Acoust Soc Am 127:2398

[3] Bergevin C, Velenovsky D, Bonine K (2010) Tectorial Membrane Morphological Variation: Effects upon Stimulus Frequency Otoacoustic Emissions. Biophys J 99:1064-1072

[4] van Dijk P, Manley GA, Gallo L, Pavusa A, Taschenberger G (1996) Statistical properties of spontaneous otoacoustic emissions in one bird and three lizard species. J Acoust Soc Am 100:2220-2227

[5] Dyson ML, Klump GM, Gauger B (1998) Absolute hearing thresholds and critical masking ratios in the European barn owl: a comparison with other owls. J Comp Physiol A 182:695-702

[6] Fischer FP, Koppl C, Manley GA (1988) The basilar papilla of the barn owl Tyto alba: a quantitative morphological SEM analysis. Hear Res 34:87-101

[7] Gummer AW, Smolders JW, Klinke R (1987) Basilar membrane motion in the pigeon measured with the Mössbauer technique. Hear Res 29:63-92

[8] Hudspeth AJ (2008) Making an Effort to Listen: Mechanical Amplification in the Ear. Neuron 59:530-545

[9] Kalluri R, Shera CA (2013) Measuring stimulus-frequency otoacoustic emissions using swept tones. J Acoust Soc Am 134:356

[10] Köppl C (1997) Frequency tuning and spontaneous activity in the auditory nerve and cochlear nucleus magnocellularis of the barn owl Tyto alba. J Neurophysiol 77:364-377

[11] Köppl C, Gleich O, Manley GA (1993) An auditory fovea in the barn owl cochlea. J Comp Physiol A 171:695-704

[12] Manley GA (2000) Cochlear mechanisms from a phylogenetic viewpoint. Proc Nat Acad Sci USA 97:11736-11743

[13] Manley GA, Schulze M, Oeckinghaus H (1987) Otoacoustic Emissions in a Song Bird. Hear Res 26:257-266

[14] Shera CA (2003) Mammalian spontaneous otoacoustic emissions are amplitude-stabilized cochlear standing waves. J Acoust Soc Am 114:244-262

[15] Shera CA, Guinan JJ (1999) Evoked otoacoustic emissions arise by two fundamentally different mechanisms: A taxonomy for mammalian OAEs. J Acoust Soc Am 105:782-798

[16] Takasaka T, Smith CA (1971) The structure and innervation of the pigeon's basilar papilla. J Ultrastruct Res 35:20-65

[17] Taschenberger G, Manley GA (1997) Spontaneous otoacoustic emissions in the barn owl. Hear Res 110:61-76

[18] Vilfan A, Duke T (2008) Frequency clustering in spontaneous otoacoustic emissions from a lizard's ear. Biophys J 95:4622-4630

[19] Wit HP, van Dijk P, Manley GA (2012) A model for the relation between stimulus frequency and spontaneous otoacoustic emissions in lizard papillae. J Acoust Soc Am 132:3273

[20] Zweig G, Shera CA (1995) The origin of periodicity in the spectrum of evoked otoacoustic emissions. J Acoust Soc Am 98:2018-2047 